\title{
LED BASED LIGHT MONITORING SYSTEM FOR THE PRIMEX EXPERIMENT AT JEFFERSON LAB
}

\author{
SAMUEL DANAGOULIAN \\ Department of Physics, North Carolina A\&T State University, \\ 1601 E. Market St. Greensboro, NC 27455 \\ E-mail: danagous@ncat.edu
}

(PRIMEX COLLABORATION)

\begin{abstract}
A multichannel Light Monitoring System (LMS) based on super-bright blue LED, has been developed for the high resolution electromagnetic hybrid calorimeter (HYCAL) at Jefferson Lab. The central part of the calorimeter consists of $1200 \mathrm{PbWO}_{4}$ crystal modules. The prototype LMS has been constructed and tested, and has demonstrated a capability of providing an adequate light pulse per each channel, equivalent to the signal of $5 \mathrm{GeV}$ electrons in the detector. The final Light Monitoring System with 1800 output channels and fiber optics light distribution system is presently under construction. The light source of the LMS represents an assembly of 31 super bright blue LEDs connected in series. The uniformity of the output light is achieved by mixing light from the LED assembly in an integrating sphere. The light intensity is being monitored using PIN photo diode and reference photomultipliers with $A m^{241}$ radioactive source. In order to achieve (0.1 - 0.3)\% long term stability, the system including the light source, the photo diode with the preamplifier and the reference PMT, is thermally stabilized.
\end{abstract}

\section{Introduction}

The PrimEx (Primakoff Experiment) ${ }^{1}$ at Jefferson Laboratory is an A- rated experiment which is designed to perform a high precision (one percent level) measurement of the partial decay width of the neutral pion in the reaction $\pi^{o} \rightarrow \gamma \gamma$. This measurement will provide a stringent test of the predictions of quantum chromodynamics in the confinement scale regime. Photons from the Hall B photon tagging system will be used to produce forward neutral pions in the Coulomb field of the target nucleus (Primakoff production). The two photons from the pion decay will be detected in the Hybrid Calorimeter (HYCAL ${ }^{2}$. The Hybrid Calorimeter has been designed to provide high precision angle and energy information for the detected photons. Angular resolution is required to cleanly distinguish the Primakoff mechanism from $\pi^{\circ}$ production from other processes, and the energy resolution will enable good invariant mass reconstruction for rejection of background neutral particles. The performance 
of HYCAL is based on high energy resolution of the central core consisting of $\sim 1200$ lead tungstate $\left(\mathrm{PbWO}_{4}\right)$ scintillating crystals. The schematic view of the detector is presented in the figure 1

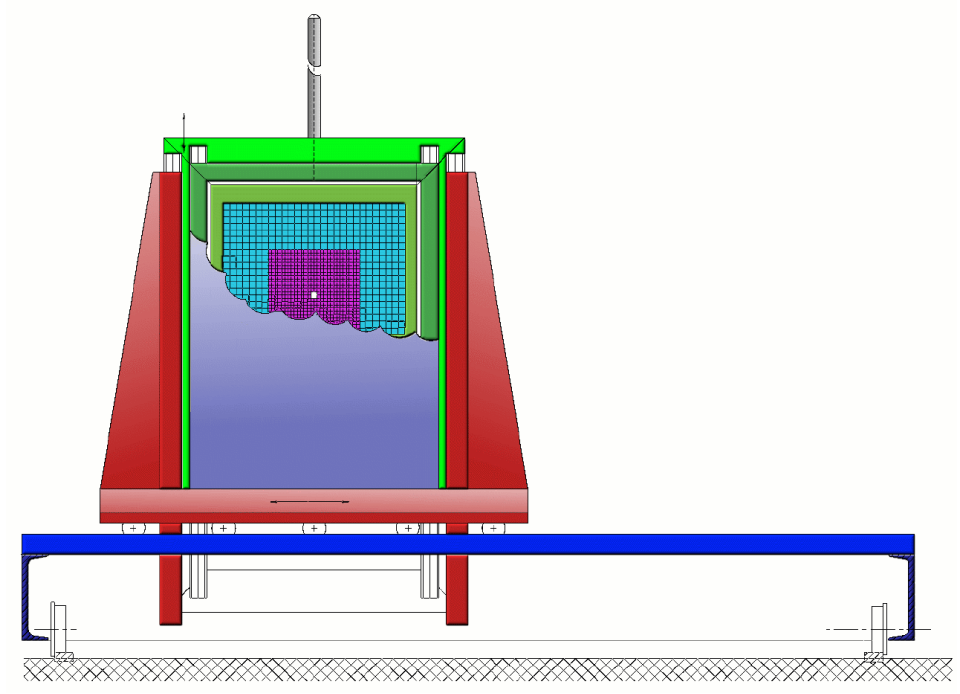

Figure 1. Schematic view of the HYCAL electromagnetic calorimeter.

In order to maintain the high performance of the calorimeter, periodic energy calibration with the tagged photon beam will be necessary. This procedure is time consuming process requiring multiple measurements with the calorimeter in different positions, and therefore cannot be done often. Consequently, an optical monitoring system will be used between successive beam calibrations as a relative calibration.

During data taking, Compton scattering events, $\gamma+" e " \rightarrow \gamma+" e "$, where "e" is an electron from the atomic orbit, might be identified among the one photon events in coincidence with one electron in the telescope of the PrimEx pair spectrometer, and might be used for calibration.

\section{PrimEx Light Monitoring System}

The main components of the Light Monitoring System (LMS) are: (1) a light source, (2) a mixing box, (3) a light distribution system, (4) reference detectors and (5) a dedicated computer to process the data for continuous stability control. The optical components and the reference detectors are mounted in a thermally insulated box whose temperature is controlled at a level of $0.1^{\circ} \mathrm{C}$. 


\subsection{The Light Source}

The light source comprises an assembly of 31 NICHIA super bright blue LEDs (peak wavelength $470 \mathrm{~nm}$ ) connected in series. The LED assembly is powered by $150 \mathrm{~V}, 8 \mathrm{nsec}$ pulses generated in a circuit, based on transistor in avalanche mode. The light pulse width is on the order of $40 \mathrm{nsec}$ on the output, which simulates the scintillation response of the lead tungstate crystal. The variation of the light pulse amplitude during a week period remains within $3.5 \%$ (see figure 2). Pulse variation over the period of one measurement (2 to $5 \mathrm{~min}$ ) is in the order of $0.2 \%$ in average. Pulse to pulse variations are negligible. The prototype light source has been continuously powered during six consecutive months and no noticeable degradation of the performance has been observed.

\subsection{Light Mixing and Distribution}

Light mixing is a necessary attribute for a high precision (sub percent level) system, as it eliminates angular and spatial variations of the input light and provides a uniform light output for further distribution. For this purpose, a four inch diameter integrating sphere has been utilized. For the final design, a second, six inch sphere will be added in order to provide $\sim 2000$ distribution channels.

Light is distributed to the individual calorimeter modules via a bundle of plastic fibers, $3 \mathrm{~m}$ long and $265 \mu$ diameter each fiber. In order to provide an adequate light pulse for each channel equivalent to the signal of $5 \mathrm{GeV}$ electrons in the calorimeter, we had to attenuate the light after the mixing chamber by a factor of about ten. The radiation degradation of light transmission in the plastic fiber will be tested during the summer-2002 upcoming beam test of the HYCAL prototype. In general, the background level in Hall B is low and the radiation damage should not be an issue. Each fiber from the bundle is terminated on the detector end by a plastic ferrule of size $5 \mathrm{~mm}$ height and $4.6 \mathrm{~mm}$ diameter, with a central hole for the fiber. The fiber tip is permanently glued inside the hole. The ferrule is then attached to the front face of the detector module. For stability of the light transmission and simplicity of attachment, the ferrules will be glued to the surface using soft UV glue (bulk modulus 200). This provides a moisture free soft interface and enables the fiber to be easily disconnected and reattached without damaging the fragile surface of the $\mathrm{PbWO}_{4}$ crystal.

\subsection{Reference Detectors}

For a stable reference detector, three types of photomultiplier tube (PMT) and a Hamamatsu PIN photo diode with low noise charge preamplifier are currently 
being tested. The reference PIN with the preamplifier is mounted directly on the light mixer. The light pulse is transmitted to the reference PMT via fiber cables from the bundle. Each reference PMT is provided with a radioactive $\alpha$-source $\left(A m^{241}\right)$ on a scintillating YAP crystal.

\subsection{Data Acquisition}

A CAMAC based Data Acquisition System with LabView DAQ software (National Instruments) is being used for the readout and on-line analysis. The signals from the reference detectors are digitized in a LeCroy 2249W (11-bit) ADC and read-out through the GPIB-CAMAC interface. The events with injected light pulse ("LMS events") will be read-out in the same way as "real events", i.e. electromagnetic shower from particles in the calorimeter module. Each type of events will have appropriate ID tag in the data stream. The monitoring system will provide a reference point through an EPICS variable every 2-3 minutes which will be included in the data stream.

The ratio of the response of each calorimeter channel to that of the LMS reference detector, for "LMS events", will be used to correct the "real data":

$$
R_{\text {corrected }}=R_{\text {raw }} \times \frac{R_{\text {light }}}{\text { Reflight }_{\text {ligh }}}
$$

Light from LMS will be periodically injected into the detector modules between real events during data taking. The frequency of light injection is defined by the optimal performance of the light source and the stability requirement for the $\mathrm{PbWO}_{4}$ crystals. On the basis of experimental tests and estimations, this frequency is chosen between 10 and $20 \mathrm{~Hz}$. A statistics of 1000-1500 "LMS events" is sufficient to determine the relative reference constant for each module. This will require 2 - 3 min data taking (in parallel with real experimental data) and will provide full relative calibration of the calorimeter.

\subsection{Results of the Stability Test}

A long term stability test of the prototype LMS has been performed and the results are presented in this section. For the test, the light intensity has been monitored with the PIN photodiode and three reference PMTs with $A m^{241}$ radioactive source each. Signals from these detectors were digitized, read-out, analyzed, and variations in time were compared with each other. The following quantities per data point of $\sim 1500$ "LMS events" are calculated for a stability factor:

$$
S_{1}=\frac{P I N}{P M T_{i}}
$$


and

$$
S_{2}=\frac{P M T_{i}}{P M T_{j}}
$$

for i-th and j-th PMTs, where $P I N$ and $P M T_{i}$ stand for the appropriate mean ADC value of the pulse height as measured with the PIN photo diode and with each PMT (the latter normalized to the average pulse height due to $A m^{241}$ source). In the figure 2 , the variation of light intensity as measured with $P M T_{2}$ and $P M T_{3}$ versus time is presented. The exposition is over the time
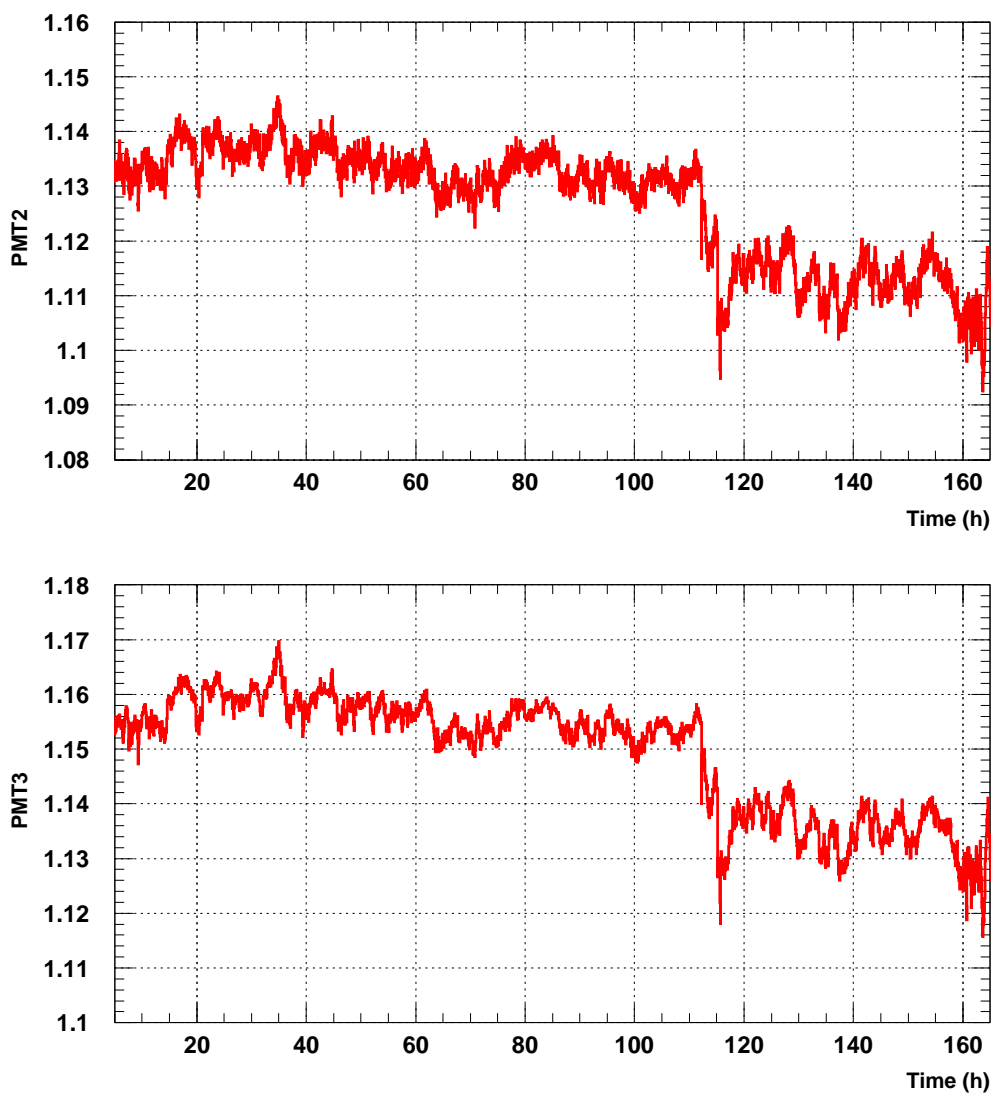

Figure 2. Variation of light intensity of the LMS vs. time 
period of 168 hours. The distribution of the stability factor, $P M T_{2} / P M T_{3}$ during the same period is shown in the figure 3 . The standard deviation of the Gaussian fit to the stability distribution is about $0.1 \%$.

\subsection{Conclusion}

A prototype Light Monitoring System for the HYCAL detector has been designed, constructed and successfully tested (in the laboratory) on the subject of stability. A stability level of $\sim 0.1 \%$ has been achieved during a week period test. A new type of light source, a group of blue LEDs connected in series and
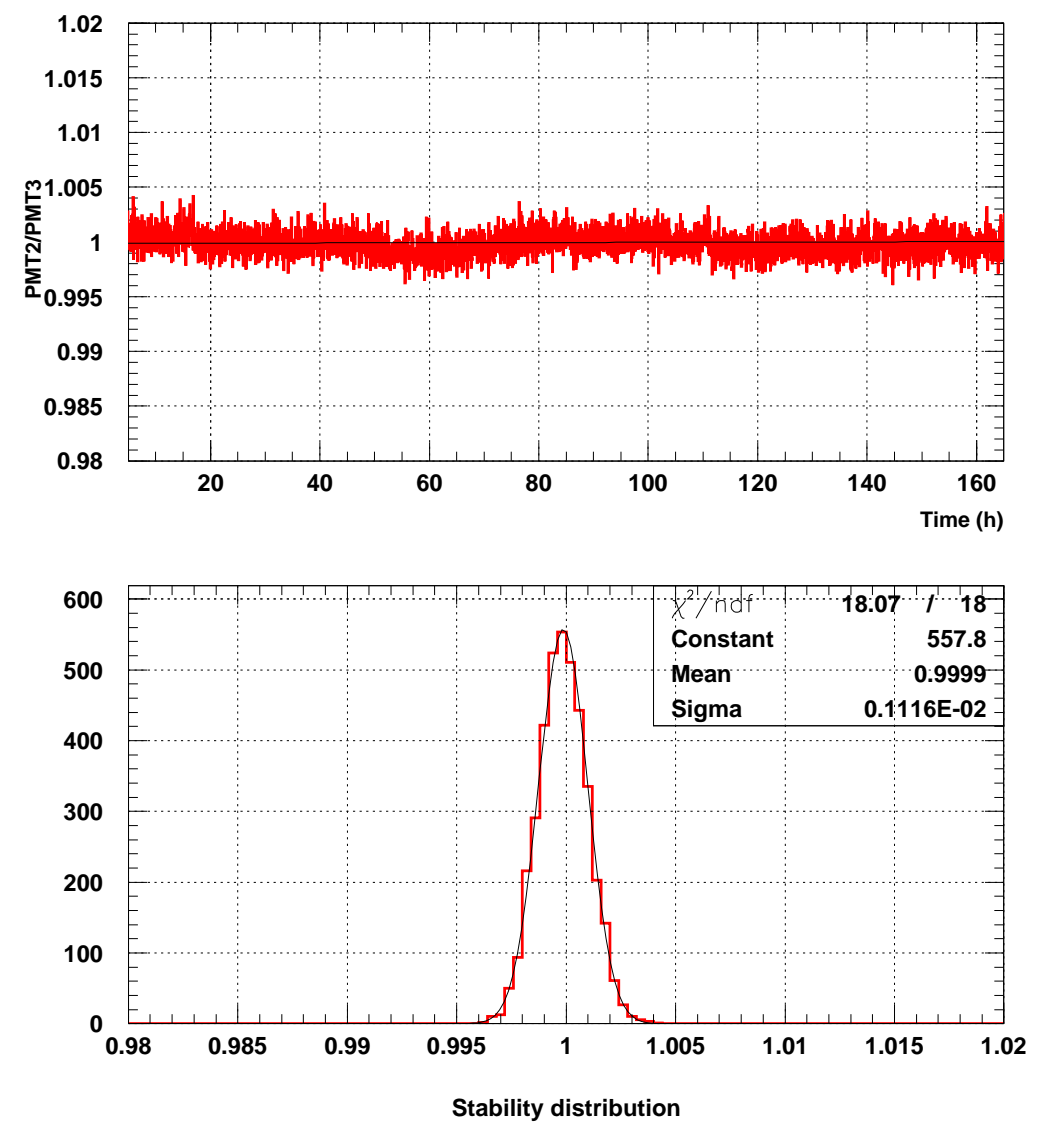

Figure 3. Stability of the light monitoring system over the period of 168 hours 
fired with a common high voltage pulse, has been developed. A long term, six months continuos run of the system, demonstrated very high reliability and good stability of the new light source.

The prototype LMS provides up to 700 channels through the fiber optics distribution system. It is ready for beam tests. The final system with $\sim 2000$ output channels is under construction and will be ready in the fall of 2002 .

\section{Acknowledgments}

This material is based upon work supported by the National Science Foundation under Grant No. 0072466 and 0079840.

\section{References}

1. D. Dale, S. Danagoulian, A. Gasparian (contact person), R. Miskimen et al., A Precision Measurement of the Neutral Pion Lifetime via the Primakoff Effect (PrimEx), Jefferson Lab Experiment E99-014 (1999)

2. Conceptual Design Report. A Precision Measurement of the Neutral Pion Lifetime via the Primakoff Effect. Jefferson Lab, March 3, 2000. 